Meta

Journal des traducteurs

Translators' Journal

\title{
Le défi du procédé synecdoquien en traduction
}

\section{Antin Fougner Rydning}

Volume 49, numéro 4, décembre 2004

URI : https://id.erudit.org/iderudit/009786ar

DOI : https://doi.org/10.7202/009786ar

Aller au sommaire du numéro

\section{Éditeur(s)}

Les Presses de l'Université de Montréal

\section{ISSN}

0026-0452 (imprimé)

1492-1421 (numérique)

Découvrir la revue

Citer cet article

Rydning, A. F. (2004). Le défi du procédé synecdoquien en traduction. Meta, 49(4), 856-875. https://doi.org/10.7202/009786ar

\section{Résumé de l'article}

La première partie du présent article traite du principe synecdoquien, un concept universel central du discours, et par la même occasion de la traduction. Je propose de relier la synecdoque de Lederer $(1976 ; 1981$ : 1994) qui consiste à voir la formulation linguistique d'un énoncé comme une partie servant à désigner un tout : le sens, à la théorie cognitive de la métaphore et de la métonymie (TCMM) (Lakoff et Johnsen 1980, Langacker 1987, Lakoff et Turner 1989, Gibbs 1994, Panther et Radder 1999 ; Barcelona 2000), où les mots servent de tremplin aux constructions cognitives. Les synecdoques sont les retombées linguistiques de l'édification d'une configuration cognitive. $\mathrm{Vu}$ qu'une notion ou une situation est en général conceptualisée différemment dans deux langues différentes, les synecdoques qui servent à véhiculer le sens d'un énoncé discursif ne sont pas les mêmes dans les deux langues. La seconde partie de l'article s'efforce de faire ressortir dans quelle mesure les traducteurs professionnels sont conscients du besoin de recourir à des synecdoques autres que celles du texte de départ en vue de restituer les configurations cognitives qu'ils ont en tête suite à la saisie du sens de formulations aussi bien innovatives que figées. Du fait que les processus mentaux ne se prêtent pas à des observations directes, force est d'inférer ceux-ci à partir des données comportementales du traducteur. Sur la base d'une expérience conduite auprès de trois professionnels de la traduction - le comportement traductionnel desquels est observé à partir de données processuelles générées par deux méthodes in vivo : les protocoles de verbalisation et le logiciel Translog - je tente d'inférer les raisons les ayant incités à opter pour des créations discursives plutôt que pour des correspondances pré-assignées. Je tente enfin de classer leurs procédures de tranfert dans trois catégories : automatique, réfléchie ou explorative.
Tous droits réservés @ Les Presses de l'Université de Montréal, 2004
Ce document est protégé par la loi sur le droit d'auteur. L’utilisation des services d'Érudit (y compris la reproduction) est assujettie à sa politique d'utilisation que vous pouvez consulter en ligne.

https://apropos.erudit.org/fr/usagers/politique-dutilisation/ 


\title{
Le défi du procédé synecdoquien en traduction
}

\author{
ANTIN FOUGNER RYDNING \\ Université d'Oslo, Oslo, Norvège \\ a.f.rydning@kri.uio.no
}

\section{RÉSUMÉ}

La première partie du présent article traite du principe synecdoquien, un concept universel central du discours, et par la même occasion de la traduction. Je propose de relier la synecdoque de Lederer $(1976 ; 1981$ : 1994) qui consiste à voir la formulation linguistique d'un énoncé comme une partie servant à désigner un tout: le sens, à la théorie cognitive de la métaphore et de la métonymie (TCMM) (Lakoff et Johnsen 1980, Langacker 1987, Lakoff et Turner 1989, Gibbs 1994, Panther et Radder 1999; Barcelona 2000), où les mots servent de tremplin aux constructions cognitives. Les synecdoques sont les retombées linguistiques de l'édification d'une configuration cognitive. Vu qu'une notion ou une situation est en général conceptualisée différemment dans deux langues différentes, les synecdoques qui servent à véhiculer le sens d'un énoncé discursif ne sont pas les mêmes dans les deux langues. La seconde partie de l'article s'efforce de faire ressortir dans quelle mesure les traducteurs professionnels sont conscients du besoin de recourir à des synecdoques autres que celles du texte de départ en vue de restituer les configurations cognitives qu'ils ont en tête suite à la saisie du sens de formulations aussi bien innovatives que figées. Du fait que les processus mentaux ne se prêtent pas à des observations directes, force est d'inférer ceux-ci à partir des données comportementales du traducteur. Sur la base d'une expérience conduite auprès de trois professionnels de la traduction - le comportement traductionnel desquels est observé à partir de données processuelles générées par deux méthodes in vivo: les protocoles de verbalisation et le logiciel Translog - je tente d'inférer les raisons les ayant incités à opter pour des créations discursives plutôt que pour des correspondances pré-assignées. Je tente enfin de classer leurs procédures de tranfert dans trois catégories: automatique, réfléchie ou explorative.

\section{ABSTRACT}

The first part of this paper discusses the synecdochial device, a key concept of a universal nature within discourse, and thus translation. I suggest linking Lederer's view of the synecdoche $(1976,1981,1994)-$, according to which the linguistic formulation is but a part used to designate the whole, i.e. the sense of an utterance - to the cognitive theory of metaphor and metonymy (CTMM) (Lakoff et Johnsen 1980, Langacker 1987, Lakoff et Turner 1989, Gibbs 1994, Panther et Radder 1999, Barcelona 2000), where words are considered as cues for cognitive constructions. The synecdoches are the overt linguistic results of an individual's ability to construct a cognitive configuration. Due to the fact that a notion or a situation is generally conceptualized differently in two different languages, the synecdoches used to convey the sense of an utterance in discourse will consequently not be the same in the two languages. The second part of this paper sets out to examine to what extent professional translators are aware of the need to resort to new synecdoches in their target text in order to convey the cognitive configurations they have in mind after having grasped the sense of both novel and entrenched linguistic expressions in the source text. As mental processes cannot be observed directly, they need to be inferred from the translator's behavioural data. Drawing on experiential cases where the professional translators' behaviour is observed on the basis of data gathered from two process-oriented on-line approaches: think-aloud-protocols and Translog key- 
board logging, I attempt to infer the reasons for their choice of translation shifts versus pre-assigned correspondences. I finally suggest classifying their transferring procedures into three categories: automatic, reflected or explorative.

\section{MOTS-CLÉS/KEYWORDS}

procédé synecdoquien, processus mentaux, conceptualisation, créations discursives versus correspondances pré-assignées, données processuelles in vivo.

\section{Introduction}

Toute traduction professionnelle est constituée d'un mélange de correspondances préassignées et de créations discursives - à savoir de formulations lexicales consignées versus des formulations lexicales non données d'avance dans la langue d'arrivée. Dans quels cas le traducteur professionnel a-t-il recours aux unes et aux autres? La production de créations discursives repose-t-elle sur des mécanismes cognitifs autres que ceux qui sous-tendent l'alignement de correspondances pré-assignées? L'activité cognitive est-elle plus importante lorsque le traducteur a affaire à une expression inédite, que lorsqu'il traduit une expression figée? L'objectif de la présente recherche', qui vise à cerner la démarche traduisante professionnelle en se fondant sur une analyse des données empiriques in vivo de trois experts-traducteurs, est d'offrir quelques éléments de réponses à ces questions.

La théorie interprétative de la traduction postule que les créations discursives sont le mode de traduction général, le niveau de transfert privilégié du traducteur soucieux de permettre au lecteur d'accéder spontanément au sens du texte et d'en assurer l'intelligibilité. L'une des raisons d'être de ces créations discursives est que le traducteur, à cheval entre deux cultures et deux systèmes linguistiques différents, ne transpose pas la synecdoque du texte de départ dans la langue d'arrivée, mais choisit une autre synecdoque pour désigner la même idée en fonction de la logique spécifique de la collectivité linguistique. Précisons que le terme de synecdoque, pris ici dans le sens qui lui est attribué par M. Lederer (1976; 1981; 1994), une des pionnières de la théorie interprétative de la traduction, n'est pas à confondre avec celui de la rhétorique classique avec lequel il n'entretient qu'un rapport marginal. Lederer a posé que les «synecdoques qu'il suffit de connaître au niveau des mots et des expressions toutes faites, doivent être créées pour établir des équivalences de textes» (1994: 59). Ce postulat théorique, selon lequel le processus créateur en traduction est engendré par le besoin de créer de nouvelles synecdoques dans le texte d'arrivée, constitue le point de départ de mon analyse de la démarche traduisante du professionnel de la traduction. Mon hypothèse de départ est que les mêmes mécanismes cognitifs sont à l'œuvre pour traduire aussi bien les expressions figées qu'inédites d'un texte.

À défaut de pouvoir accéder directement à l'activité cérébrale déployée par le traducteur lors de la compréhension et la formulation des énoncés discursifs du texte, force est de recourir, pour la description de la démarche traduisante, à des méthodes d'observation indirecte de la façon dont les experts-traducteurs s'acquittent de leur tâche. Dans le cadre de la présente étude, les deux méthodes d'enregistrement in vivo retenues pour capter leurs réflexions, leur comportement, leurs activités d'écriture, celles-ci comprenant les corrections et révisions effectuées, ainsi que les pauses observées pendant l'opération traduisante, sont l'approche TAPs (Think-AloudProtocols) et l'approche Translog (voir section 2. Méthodes de collection des données 
processuelles). À partir des données que ces deux méthodes génèrent, je suggère de cerner de plus près la façon dont ils s'acquittent de leur tâche face à la traduction de locutions aussi bien figées qu'inédites.

Récapitulons: Dans un premier temps, je rends compte du phénomène de la synecdoque tel qu'il est conçu par Lederer $(1976$; 1981 ; 1994) tout en le rapprochant du procédé conceptuel métonymique des cognitivistes (Lakoff et Johnsen 1985, Langacker 1987, Lakoff et Turner 1984, Gibbs 1994, Gibbs et Steen 1999, Panther et Radder (eds) 1999, Barcelona (ed.) 2000) - celui-ci constituant avec le procédé conceptuel métaphorique une théorie au fort pouvoir explicatif: la théorie cognitive de la métaphore et de la métonymie (voir Rydning 2002 et 2005 sous presse) - avant de montrer combien est pertinente pour la traduction le procédé synecdoquien. Je présente dans un deuxième temps les méthodes de collection des données TAPs et Translog sur lesquelles je fonde dans un troisième temps, avec les données textuelles, mon analyse. Je montre enfin quelles sont les procédures de transfert adoptées par les sujets traduisants au moyen de trois exemples extraits de mon corpus.

\section{Le procédé synecdoquien appliqué à la traduction}

\subsection{La synecdoque ledererienne et le procédé métonymique des cognitivistes}

La métonymie, la synecdoque et la métaphore sont généralement associées aux effets de style dans les textes poétiques et littéraires. Traditionnellement conçues comme un procédé référentiel ornemental, elles ont comme dénominateur commun le fait d'établir un lien entre deux choses. Ainsi dans les exemples ci-dessous empruntés à Reboul (1994: 127-128):

(1) Le trône et l'autel

le «trône» réfère métonymiquement à la puissance du roi, et l'«autel» à celle de l'Église.

Dans:

(2) Cent têtes

le terme «têtes» réfère par voie de synecdoque à personnes. Dans:

(3) Sophie est un glaçon

«glaçon» renvoie métaphoriquement à la froideur en amour de Sophie.

S’inspirant de la caractéristique consistant à établir un lien entre deux choses, Lederer emprunte à la rhétorique classique le terme de «synecdoque» dans son étude pionnière Synecdoque et traduction (1976) et l'applique à la traduction. Elle confère cependant à cette figure de style une propriété conceptuelle, et démontre que le principe de désignation au niveau de la langue, consistant à prendre un aspect caractéristique d'un concept pour transmettre la totalité du concept, est également applicable au niveau du discours, où un trait saillant d'une idée sert à transmettre l'idée entière. Lederer observe que "ce procédé est sans cesse appliqué, de manière parfaitement normale et tout à fait inconsciente par tous les locuteurs dans tous les types de discours» (1976: 14). La synecdoque ledererienne est donc un procédé qui sert à référer à un tout au moyen de la partie. Cette référence peut être établie aussi bien au sein d'un même domaine conceptuel (métonymie et synecdoque), que d'un domaine conceptuel à un autre (métaphore). Par rapport à la rhétorique classique 
qui établit une distinction entre la métaphore et la métonymie d'une part, et la métonymie et la synecdoque d'autre part, la synecdoque - prise dans le sens qui lui est attribué par Lederer - que j'adopte, englobe les trois types de figures sans opérer de distinction, celle-ci au demeurant difficile à établir (voir Barcelona 2000; Rydning 2003), n'étant pas pertinente en traduction. Or, pour éviter toute confusion avec la figure de style de la rhétorique classique, je retiens le terme de procédé synecdoquien pour renvoyer au procédé de désignation de l'ensemble d'une notion ou d'un phénomène par le biais d'un de ses aspects, actualisé par le discours.

Les travaux cognitivistes sur la métonymie (Lakoff et Johnsen 1985, Lakoff et Turner 1984, Gibbs 1994, Barcelona (ed.) 2000, Panther et Radden (eds) 1999) reposent sur une conception analogue à celle de Lederer. L'expression linguistique évoque une structure conceptuelle, laquelle fournit un accès mental à une autre entité conceptuelle. Mais au lieu de retenir le terme de synecdoque, les cognitivistes choisissent celui de métonymie et, à l'instar de Lederer, ils l'accommodent par rapport à la définition rhétorique classique pour renvoyer au procédé qui permet de référer à un objet ou un concept en désignant une partie de cet objet ou de ce concept. Pour eux, la métonymie fait partie intégrante, avec la métaphore, des systèmes conceptuels quotidiens qui sous-tendent notre façon de penser et d'agir.

[...] like metaphors, metonymic concepts structure not just our language but our thoughts, attitudes, and actions. (Lakoff et Johnsen 1984: 39)

\subsection{Le procédé synecdoquien, concept clé en traduction}

Les différentes langues ne choisissent pas les mêmes aspects pour désigner les mêmes concepts. Il suffit de se reporter aux adages dans deux langues différentes pour constater qu'ils désignent le même concept au moyen de mots différents. Ainsi porter de l'eau au moulin correspond en anglais à to bring coals to Newcastle. Bien que les images qu'évoquent ces deux expressions soient différentes, elles renvoient toutes deux au fait de donner involontairement à quelqu'un des arguments dans un débat.

C'est à Lederer que revient le mérite d'avoir la première remarqué les retombées du procédé synecdoquien sur la traduction. Tout comme chaque langue choisit différemment les traits saillants qui servent à désigner objets et concepts, les idées dans le discours sont évoquées au moyen de certains traits propres à la langue d'arrivée. Traduire en se contentant de transposer les traits saillants de la langue de départ risque non seulement de nuire à l'intelligibilité de la traduction, mais plus grave encore, de fausser le sens. Le remède suggéré pour rendre justice au sens exprimé dans l'énoncé original est d'actualiser les traits saillants de la langue d'arrivée en fonction de la logique d'expression de celle-ci. Une question qu'il convient de se poser à cet égard, est celle de savoir quels traits saillants actualiser en vue de restituer intelligiblement le sens compris. La présente étude vise à fournir quelques éléments de réponse à cette question en se fondant sur les données textuelles et processuelles des sujets traduisants. Comme une analyse des seules données textuelles ne permet pas toujours d'inférer les raisons qui ont incité le traducteur à retenir tels traits saillants plutôt que tels autres, je prends appui aussi sur les données processuelles des sujets traduisants. 


\section{Méthodes de collection des données processuelles}

\subsection{Méthodes générant des données in vivo}

Partant du principe que le comportement des sujets traduisants sert d'indice à leur activité cognitive (Jakobsen 2000: 157, Jensen 2000: 97), deux méthodes d'observation in vivo des sujets traduisants ont été combinées, à savoir l'approche qualitative dite TAPs (Think-Aloud Protocols) et l'approche informatisée des activités d'écriture et des pauses Translog.

1. L'approche introspective TAPs où les sujets traduisants, laissés seuls dans leur bureau, pensent à haute voix pendant qu'ils traduisent. Les données verbales qui en émanent sont enregistrées sur vidéo et transcrites en protocoles de verbalisation (Voir Ericsson et Simon 1980, 1993, Tirkkonen-Condit 1989, 1992, Jääskelaäinen 1987, 1989, 1990, 2000).

2. L'approche informatisée des activités d'écriture des sujets traduisants dite Translog, programme développé par Jakobsen et Skou (1999). Ce logiciel enregistre toutes les données correspondant aux:

- touches du clavier de l'ordinateur;

- pressions sur l'interrupteur de la souris;

- temps d'arrêt marqués entre les périodes d'écriture.

Ces données peuvent être représentées graphiquement ainsi qu'en différé. La fonction play-back permet de visualiser l'opération traduisante en temps réel, accéléré ou réduit (Voir Jakobsen 1999, 2000).

\subsection{Nature des données du corpus}

Les données TAPs et Translog qui servent de point de départ à la présente étude sont fournies par trois experts-traducteurs, lesquels ont été priés de traduire dans leur langue $\mathrm{A}$, le norvégien, le texte original français reproduit ci-après, pour publication dans le quotidien norvégien Dagens Noeringsliv, tout en verbalisant leurs pensées.

(4) Le retour des bonnes manières

On les croyait désuètes, balayées par l'ouragan post-soixante-huitard, promises à une mort imminente par la recrudescence des incivilités. On les imaginait figées, tout juste bonnes à illustrer les manuels d'éducation pour jeunes filles de bonne famille. Erreur. Liftées, épurées, les bonnes manières sont à nouveau plébiscitées par les Français. Les bonnes manières sont un signe d'intégration. Mais elles restent aussi, plus sournoisement, un mode de tri social. Raison de plus pour maîtriser les codes. Les entreprises exigent aujourd'hui de leurs employés qu'ils sachent communiquer. Le «BSAM» (bonjour-sourire-au revoir-merci) est enseigné partout. Conseillère en image personnelle, Hélène Choumiloff veille au grain. Ses «élèves» sont des cadres de sexe masculin de plus de 40 ans. Leur objectif: éviter les gaffes au cours des dîners d'affaires. «Beaucoup de mes clients se sont faits à la force du poignet. C'est l'ascension sociale qui crée la gêne», constate la conseillère. Pendant les exercices, dans la rue ou au restaurant, elle traque les failles. L'apprentissage peut durer trois mois. Les élèves sont très motivés. S'il est désormais bien vu de laisser au placard cravate et costume trois pièces le vendredi, nul ne tolère, désormais, les infractions au code de bonne conduite. 
La façon la plus économique d'accéder au processus de la traduction est l'étude de la performance d'experts en traduction (Tirkkonen-Condit 2002: 11). Ce qui distingue l'expert du non-expert est la supériorité de sa performance. L'expert sait comment s'y prendre pour résoudre des problèmes difficiles, ses décisions sont en général rationnelles et motivées. Un critère d'expertise en traduction est un minimum de 10 000 heures d'expérience active de la traduction professionnelle (Posner 1988: xxxi, cité par Jääskeläinen 2003). Ce critère rejoint celui proposé par Shreve (2002: 161), selon lequel on ne devient expert que moyennant au moins 10 ans de pratique délibérée. Ces critères m’ont amenée à sélectionner les participants experts à la présente étude avec la plus grande précaution. J'ai recruté trois experts-traducteurs agréés par l'État norvégien ${ }^{2}$ attestant 15 ans d'expérience active de la traduction professionnelle, considérées comme les meilleures dans la profession. Toutes les trois sont diplômées de l'ESIT, et ont en outre travaillé en parallèle comme interprètes de conférence. Ces trois experts-traducteurs sont désignés ci-après par les noms fictifs suivants: Anne, Brigitte et Christine.

Les données du corpus comprennent, d'une part, des données textuelles, à savoir le texte original français et ses trois traductions en norvégien et, d'autre part, des données processuelles in vivo, à savoir les commentaires verbaux de chacun des trois experts-traducteurs aux prises avec leur texte, ainsi que leurs activitiés d'écriture et les pauses enregistrées par le logiciel Translog. Les données processuelles génèrent trois types de données reliées entre elles sur lesquelles je prends appui pour proposer une classification des procédures de transfert des experts-traducteurs. Il s'agit de données linguistiques, temporelles et comportementales à partir desquelles il est possible d'identifier un certain nombre d'indices susceptibles de refléter les activités mentales du traducteur.

\subsubsection{Données linguistiques}

Par données linguistiques, j'entends, dans l'enregistrement Translog les signes graphiques, c.à-d. les chiffres et les lettres qui constituent des mots ou des débuts de mots se situant de part et d'autres des pauses du texte, ces dernières étant représentées par *, ainsi que les corrections et révisions apportées aux segments écrits du texte marquées par $凶$. Donnons en exemple la solution provisoire (6) ci-après extraite des données Translog de Christine, expert-traducteur, en vue de traduire l'énoncé (5) ci-dessous, suivie de ma traduction française (7) où les solutions provisoires sont mises entre parenthèses :

(5) On les croyait désuètes, balayées par l'ouragan post-soixante-huitard [...]

(6) Données Translog de Christine

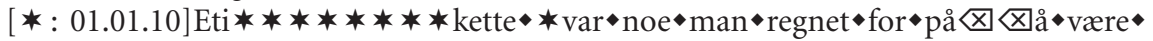

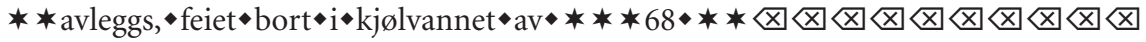

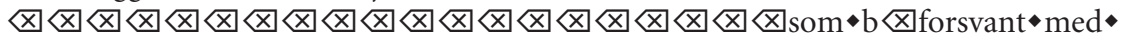
$69 \otimes 8 \bullet$ generasjonen

(7) Le bon usage était considéré être désuet (, balayé dans le sillage de) et a disparu avec la génération de (69) 68

Nous notons que le premier terme «Etikette» n'est pas aligné d'un trait. Une pause de huit secondes suit l'inscription des trois premières lettres. La première correction porte sur une faute d'orthographe: «på». Ce mot est effacé, puis remplacé par «å » 
(la marque de l'infinitif en norvégien). La deuxième correction porte sur «feiet bort i kjølvannet av»: balayés dans le sillage de, au profit de «som forsvant med»: qui ont disparu avec. La troisième correction porte sur le chiffre 9 , remplacé par 8 pour former 68.

Dans les protocoles de verbalisation les données linguistiques sont constituées par la transcription des commentaires verbaux des experts-traducteurs. Ces commentaires verbaux peuvent avoir trait aux stratégies tant globales que locales du traducteur et porter sur divers aspects du processus de traduction, tels la reconnaissance d'un problème linguistique ou référentiel, la volonté de combler l'écart entre la méconnaissance référentielle et la connaissance factuelle jugée nécessaire pour saisir le sens de l'énoncé, la prise en considération de facteurs communicationnels dans la recherche d'une solution donnant accès au sens, la conformité aux normes de la langue d'arrivée, le souci d'adapter le texte d'arrivée aux destinataires.

\subsubsection{Données temporelles}

Par données temporelles j'entends les pauses qui se situent de part et d'autre des activités d'écriture dans les données Translog qui sont indiquées par un ou plusieurs *, chaque * correspondant ici à une pause d'une seconde, ainsi que par des indications entre crochets correspondant à la durée des pauses, comme:

(8) [*: 41.39], à savoir une pause de 41.39 secondes.

J'ai écrit ailleurs (Rydning 2004) que les pauses signalent un effort cognitif. Une étude des pauses dans la production routinière de textes effectuée par Joost Schilperoord corrobore l'idée selon laquelle les pauses sont révélatrices d'activités mentales: "the time interval between the presentation of a stimulus and the production of a response is taken to be a measure of the amount of cognitive processes the subject needs for his response» (Schilperoord 1996: 18). Plus les pauses sont longues, plus l'effort cognitif fourni pour résoudre le problème en question semble soutenu. D'après Jakobsen les pauses de plus de 10 secondes sont des signes d'un effort cognitif particulier: "With most translators, and in most situations, a time value greater than ten seconds will tend to identify text-initial and text-final delays, delays between paragraphs, and delays appearing less systematically before text segments that are particularly difficult to translate» (Jakobsen $2000: 167$ ).

\subsubsection{Données comportementales}

Les données comportementales reflètent:

a. Les activités mentales généralement associées à la tâche de traduction, telles la lecture, l'écriture, la réflexion, la relecture, les corrections, la consultation de dictionnaires, la révision.

b. Les états d'âme des sujets traduisants qui se manifestent au niveau des données verbales/auditives sous forme d'interjections, d'emphase, de jurons, de soupirs de frustration ou de soulagement, de rire, comme:

(9) Voilà, c'est ça! (Interjection en français chez Frédéric)

(10) Klatringen, ja! (Irène) (C'est ça, s'élever!)

(11) Merde! (Juron en français chez Frédéric) 
Au niveau des données non verbales/non auditives, les états d'âme des sujets traduisants se manifestent au moyen de gestes subconscients, tels se gratter la tête, pianoter, se taper le ventre, sucer son crayon; de gesticulation, comme les mouvements des mains qui simulent le fait de balayer, de croître ou qui, par imitation, précède une expression imagée, comme ouvrir les bras pour signaler le sens de «souhaiter la bienvenue» pour traduire le terme indiqué en caractères gras dans l'énoncé original suivant: "Liftées, épurées, les bonnes manières sont à nouveau plébiscitées par les Français.»; d'expressions faciales subconscientes, comme sourire, froncer les sourcils, grimacer, se mordre la lèvre; et de mouvements qui ne sont en général pas associés à la tâche de traduction, comme regarder par la fenêtre, se lever, à la suite de quoi, très souvent, les sujets traduisants produisent une solution provisoire ou finale à un problème de transfert (voir à ce sujet le cas de Christine reporté au ci-dessous).

c. Les activités mentales qui ne se recoupent pas nécessairement avec celles annoncées dans les commentaires verbaux. À la recherche d'une formulation linguistique susceptible de restituer l'idée bien comprise dans la synecdoque un mode de tri social, pour laquelle Christine a retenu la solution provisoire skille "séparer», elle décide de s'inspirer de son dictionnaire unilingue norvégien. Or, son téléphone sonne au moment où elle avance le bras pour l'ouvrir, et elle se lève pour répondre. Aussitôt la conversation achevée, elle reprend son dictionnaire qu'elle feuillète sans toutefois le consulter, une solution s'étant entre-temps présentée à elle: sortere «classer». Ce n'est donc pas son dictionnaire qui lui fournit la solution provisoire - au demeurant fort éloignée de celle qu'elle finira par adopter. Celle-ci est le fruit d'une inspiration s'étant présentée à elle après un temps d'arrêt où sa concentration a été interrompue par une activité n'ayant rien à voir avec la tâche de traduction. Parfaitement consciente de ce phénomène, Christine observe que l'inspiration lui vient souvent après s'être détendue ou avoir fait quelques pas dans son bureau: "Se détendre et faire quelques pas est souvent utile - je recours souvent à ce procédé.»

\section{Analyse des données textuelles et processuelles des experts-traducteurs}

Comparons dans un premier temps les locutions de l'original aux solutions finales retenues dans la langue d'arrivée pour chacun des sujets traduisants dans les trois énoncés ci-après extraits du texte Le retour des bonnes manières, et faisons la part entre les créations discursives et les correspondances pré-assignées.

(12) On les croyait désuètes, balayées par l'ouragan post-soixante-huitard, promises à une mort imminente par la recrudescence des incivilités.

(13) Le «BSAM» (bonjour-sourire-au-revoir-merci) est enseigné partout.

(14) Beaucoup de mes clients se sont faits à la force du poignet.

Rendons compte dans un deuxième temps des données processuelles in vivo des experts-traducteurs face aux locutions aussi bien figées qu'inédites auxquelles ils sont confrontés. 


\section{1 [...] balayées par l'ouragan post-soixante-huitard [...]}

En termes cognitivistes, il y a interaction entre la métaphore conceptuelle de l'ouragan qui ravage tout sur son passage et la métonymie conceptuelle où «soixantehuitard» réfère à l'esprit des idéaux libertaires de la génération 68 .

\subsubsection{Comparaison de l'énoncé (12) avec les solutions retenues par Anne (15),} Brigitte (16) et Christine (17), suivies de ma traduction, laquelle se veut aussi proche de l'original que possible sur le plan lexical, sans pour autant violer le respect au maniement du français:

(15) Anne:

En trodde de gamle gode manérer var gått av moten, at sekstiåtte-generasjonen hadde feid dem av banen [...]

On croyait que les vieilles bonnes manières étaient passées de mode, que la génération soixante-huit les avait balayées du pont [...]

(16) Brigitte:

Mange trodde at gode manerer var passé, at de hadde blåst bort med vinden etter seksiåtterne $[\ldots]$

D'aucuns croyaient que les bonnes manières n'étaient plus, qu'elles avaient été emportées par le vent après les soixante-huitards [...]

(17) Christine:

Etikette var noe man regnet for å være avleggs, noe som ble feid bort $i$ kjølvannet av 68 revolusjonen $[\ldots]$

Les usages étaient considérés désuets, balayés dans le sillage de la révolution 68 [...]

Nous constatons que la métaphore de l'ouragan véhiculant l'idée de faire table rase n'a été conservée dans aucune des trois créations discursives. La solution qui se rapproche le plus de l'original est celle de Brigitte (16), où le terme «vind » : vent dans la locution figée «blåst bort med vinden»: emportées par le vent, sert à évoquer la notion de disparition. Dans la solution d'Anne (15), c'est la notion de déblaiement qui est présente à l'esprit: nous visualisons les soixante-huitards à l'œuvre avec leurs balais pour faire le propre. Dans la solution de Christine (17), la notion de nettoiement est perçue. Les images évoquées par ces trois traductions sont différentes, mais renvoient (plus ou moins clairement) à la notion de se débarrasser de quelque chose d'encombrant. Le recoupement conceptuel avec l'original est évident. Les différences retenues se situent au niveau de la force exercée. Nous visualisons une force ravageuse dans l'énoncé original, alors qu'elle est nettement plus faible dans les trois traductions.

\subsubsection{Analyse des données processuelles}

Dans quelle mesure les données processuelles permettent-elles de déceler les raisons qui ont incitées les experts-traducteurs à choisir des traits saillants autres que ceux de l'original pour réexprimer l'idée? Par manque de place, je me vois contrainte ici de ne reporter que les données processuelles d'un expert-traducteur par énoncé. 
(18) Commentaires verbaux relevés dans le protocole TAPs d'Anne

\begin{tabular}{|l|l|}
\hline Commentaires verbaux d'Anne & Traduction française \\
\hline $\begin{array}{l}\text { Det gjelder jo liksom ... å komme ... inn } \\
\text { i tankegangen her (ser på papirversjonen }\end{array}$ & Il s'agit en quelque sorte de comprendre \\
av originalteksten) for å få litt sveis... & la façon de penser ici (regarde la version \\
sving på det. (Tenker) (Skriver) (Sukker) & papier du texte original) pour trouver \\
une forme seyante ...qui sonne bien. \\
(Mumler det da snakk om sekstiåtterne. & (Réfléchit) (Écrit) (Soupire) Il s'agit bien \\
& ici des soixante-huitards. (Murmure) \\
& (Réfléchit) (Écrit). \\
\hline
\end{tabular}

Anne fonde manifestement son inspiration sur une bonne compréhension du texte. Après un temps de réflexion assez long (20,78 secondes d'après ses données Translog voir ci-après) pause pendant laquelle elle cherche visiblement à comprendre la notion évoquée par «l'ouragan post soixante-huitard» elle fait fusionner «ouragan » et «soixante-huitard». Cette fusion conceptuelle permet de visualiser l'ouragan sous la forme de personnes - procédé qui a pour effet de doter les soixante-huitards de caractéristiques propres à l'ouragan, notamment celle de tout emporter sur leur passage. Voyons à présent si les données Translog d'Anne peuvent nous fournir une explication sur les motifs qui l'ont incitée à composer une synecdoque inédite.

(19) Les données Translog d'Anne

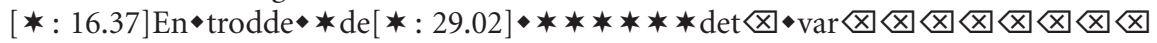
de var gått $\bullet \bullet$ moten $*$, at $* * * * *$ de $[*: 20.78]$ seksti $*$ åtte**|凶generasjonen $* * * * * * * * * * *$ haddew $* * * * * * *$ feid $\bullet$ dem $\bullet$ av banen $* * * *$

(20) Traduction où les solutions provisoires sont mises entre parenthèses:

On croyait (que les) (qu’il était) (qu'elles étaient démodées), (que les) soixantehuit(ards)/ que la génération les avait balayées du pont

(21) Traduction corrigée, abstraction faite des solutions provisoires:

On croyait que la génération soixante-huit les avait balayées $d u$ pont

La pause de 20,78 secondes qui précède l'alignement de «soixante-huitards » est suivie d'une nouvelle pause de 2 secondes, à la suite de laquelle Anne inscrit une barre oblique, indice d'une solution alternative se présentant à l'esprit du sujet traduisant (voir Rydning 2004), qu'elle efface cependant aussitôt. Elle tape ensuite "génération» avant de marquer une nouvelle pause de 11 secondes. Ses données Translog montrent qu'en optant pour une solution légèrement plus explicite dans sa traduction: la génération soixante-huit, que celle évoquée dans ses commentaires verbaux: les soixante-huitards, elle avalise son idée initiale. Au lieu d'écrire "soixante-huitards", solution qui réfère par métonymie à la notion abstraite d' "esprit des idéaux libertaires de la génération 68 », elle retient le terme plus concret de "génération", lequel sera doté de l'épithète «soixante-huit», solution synonyme qui réfère par métonymie à la même notion.

\subsubsection{Analyse des données d'Anne}

Les propos déclaratifs d'Anne dans (18) sont révélateurs de la stratégie globale de traduction qu'elle adopte: Elle est confiante de pouvoir trouver une forme linguistique adéquate dans la langue d'arrivée susceptible d'évoquer l'idée de l'énoncé original, 
une fois le sens compris. Grâce à ses données linguistiques et comportementales, nous sommes à même de retracer son cheminement cognitif. Aussitôt après avoir établi un lien conceptuel entre "ouragan» et "soixante-huitard», Anne crée une nouvelle synecdoque. Bien que (22) et (23) ci-dessous fassent état d'un certain recoupement lexical (voir les termes en caractères gras), il semble logique d'inférer que la nouvelle synecdoque est fonction de la scène de déblaiement: les soixante-huitards qui nettoient le pont à l'aide de leurs balais, et non de la formulation linguistique originale.

(22) $[\ldots]$ balayées par l'ouragan post-soixante huitard [...]

(23) [...] la génération soixante-huit les avait balayées du pont [...]

\section{$3.2 \mathrm{Le}$ «BSAM»}

Passons maintenant à l'exemple de la locution inédite: le «BSAM» tel qu'il apparaît dans l'énoncé (13): Le "BSAM» (bonjour-sourire-au-revoir-merci) est enseigné partout. Insérée dans le texte (4) Le retour des bonnes manières, l'abréviation «BSAM » désigne un sens plus vaste que son sémantisme (les quatre marques de politesse), car elle renvoie par voie de synecdoque à un critère de comportement social courtois exigé par les entreprises. La référence au tout - en l'occurrence le domaine conceptuel du code de bonne conduite - est établie dans le texte au moyen de la juxtaposition des traits saillants de politesse: bonjour-sourire-au-revoir-merci.

\subsubsection{Comparaison de l'énoncé (13) aux solutions retenues par Anne (24), Brigitte (25) et Christine (26):}

(24) Anne:

A kunne si goddag, adjø, takk og å smile på riktig vis, er noe som det undervises i overalt. Pouvoir dire bonjour, adieu, merci et sourire tout en respectant les convenances, font partie de ce qui est enseigné partout.

(25) Brigitte :

Det undervises i 'GSAT' (goddag-smil-farvel-takk) overalt.

Le 'BSAM' (bonjour-sourire-prendre congé-merci) est enseigné partout.

(26) Christine:

Kurs i skikk og bruk tilbys overalt.

Des cours de savoir-vivre sont offerts partout.

Nous constatons qu'Anne a choisi de faire abstraction du sigle dans sa traduction. Elle opte pour deux constructions infinitives qu'elle coordonne: (i) "pouvoir dire bonjour, adieu, merci» et (ii) 'et sourire', auxquelles elle rajoute: «tout en respectant les convenances». Par cet ajout, Anne s'efforce de restituer la notion implicite du comportement social courtois exigé par les entreprises. Brigitte module la formulation linguistique originale, mais ne transpose pas correctement l'initiale de la troisième marque de politesse: «farvel», ce qui fait apparaître une légère incohérence. Sa traduction peut être qualifiée de correspondance pré-assignée. À la différence d'Anne et de Brigitte qui ont opté pour le transfert de chacun des traits saillants de politesse contenus dans BSAM, Christine a choisi de restituer l'idée contenue dans l'expression inédite par un aspect plus global: «skikk og bruk»: le savoir-vivre. Cette décision a fait l'objet d'une mûre réflexion, suivie de commentaires dans son protocole 
de verbalisation sur l'inutilité de reproduire la façon française de présenter l'idée dans sa traduction. Examinons à présent de plus près les données processuelles de Christine.

\subsubsection{Analyse des données processuelles de Christine}

(27) Commentaires verbaux relevés dans le protocole TAPs de Christine

\begin{tabular}{|c|c|}
\hline Commentaires verbaux de Christine & Traduction française \\
\hline $\begin{array}{l}\text { Bonjour-sourire-au-revoir-merci. Ja, det } \\
\text { er altså disse BSAM. Bon chic, bon genre. } \\
\text { Det tror jeg ikke har noen hensikt å } \\
\text { beholde den. Skikk og bruk. Ja, jeg tror vi } \\
\text { tar rett og slett skikk og bruk. Skikk og } \\
\text { bruk blir, skal vi se... (Tenker) Kurs, } \\
\text { kanskje? Her er jeg ikke sikker på skikk } \\
\text { og bruk. Kurs i skikk og bruk kanskje? Ja, } \\
\text { dette var jo... Jeg tror kanskje jeg } \\
\text { beholder den. }\end{array}$ & $\begin{array}{l}\text { Bonjour-sourire-au-revoir-merci. Voici } \\
\text { donc ces BSAM. Bon chic, bon genre. À } \\
\text { mon avis, il ne sert à rien de le garder. Le } \\
\text { savoir-vivre. Oui, je pense carrément } \\
\text { mettre le savoir-vivre. Le savoir-vivre est, } \\
\text { voyons voir. Des cours, peut-être? Je ne } \\
\text { suis pas sûre ici pour savoir-vivre. Des } \\
\text { cours de savoir-vivre, peut-être? Eh oui, } \\
\text { c'est bien... Je crois que je vais la garder. }\end{array}$ \\
\hline
\end{tabular}

Après avoir établi une analogie avec le sigle conventionnel français: BCBG, Christine écarte d'emblée la solution consistant à moduler les initiales qui forment le sigle inédit BSAM en norvégien. Bien que Christine ne fournisse pas d'explication à ce choix délibéré, il semble que ce soit les normes du norvégien qui l'incitent à choisir la création discursive: "le savoir-vivre». Alors que les sigles et les acronymes pullulent dans les textes français, ils sont plutôt rares en norvégien. Le sigle inédit du texte original est un excellent exemple du penchant qu'ont les Français d'en créer spontanément au fil de leurs pensées. Les Norvégiens en sont, quant à eux, peu friands: ces modes de constitution de mots étant beaucoup moins productifs qu'en français. Nous notons aussi que Christine part sur l'idée de transférer la tournure passive du français en norvégien: "Le savoir-vivre est», mais après une courte hésitation, elle propose d'insérer 'des cours de' devant le syntagme nominal. Après avoir "goûté» à cette solution, elle lui donne sous réserve son aval. La description ci-après des données Translog de Christine permet de mieux comprendre son hésitation.

(28) Les données Translog de Christine

$* * * *$ Skikk og $\bullet$ bruk blitr $\otimes \otimes \otimes \otimes \otimes * * * \otimes \otimes * * * * *$ kurs $\bullet * \leftarrow \leftarrow \leftarrow \leftarrow \leftarrow$ $\bullet \rightarrow \rightarrow \rightarrow \rightarrow \cdot * \leftarrow \leftarrow \leftarrow \leftarrow \otimes \otimes \otimes \otimes \otimes \otimes \otimes \otimes \otimes \otimes \otimes \otimes \otimes \otimes \otimes K \rightarrow \rightarrow \rightarrow \bullet i \bullet$ skikk og bruk tilbys overalt.

(29) Traduction où les corrections effectuées sont mises entre parenthèses (Le savoir-vivre est) (d)es cours D sur le savoir-vivre sont offerts partout.

(30) Traduction corrigée

Des cours sur le savoir-vivre sont offerts partout.

Après une courte pause de quatre secondes, Christine aligne "Skikk og bruk blir»: Le savoir-vivre est, mais efface aussitôt ce début de tournure passive, ressentant sans doute que le verbe transitif direct «undervise» (enseigner) au participe passé ne se prête pas dans le contexte du sujet abstrait «skikk og bruk» (savoir-vivre) à la passivation avec le verbe «bli»(devenir). Plutôt que de recourir à une construction 
impersonnelle, Christine opte pour une variante de la tournure passive, dite «spassiv» en norvégien ${ }^{3}$.

\subsubsection{Analyse des données de Christine}

J'interprète la pause Translog relativement longue de 23,73 secondes au cours de laquelle Christine marque un temps d'arrêt avant de commenter le BSAM, ainsi que ses commentaires verbaux sur l'inutilité de créer un sigle correspondant en norvégien, comme des indices d'un problème de reformulation qu'elle résoud au moyen d'une création discursive. Christine n'éprouve visiblement aucune difficulté à saisir le sens véhiculé par BSAM. Bien au contraire, nous avons vu qu'elle établit spontanément une analogie avec BCBG. Si elle n'a pas eu besoin ici de recourir à des solutions intermédiaires avant de proposer sa solution finale, il est néanmoins intéressant de situer celle-ci par rapport aux trois solutions provisoires suggérées plus tôt au sujet de l'expression synonyme bonnes manières rencontrée dans le titre du texte:

(31) gode manérer - «bonnes manières"

(32) etiquette - «étiquette»

(33) skikk og bruk - «savoir-vivre»

Christine ne retient aucune de ces solutions intermédiaires et décide de reporter la traduction du titre à plus tard. Or, lorsque le syntagme bonnes manières réapparaît sous sa forme pronominalisée les dans la première phrase du texte, elle se sent contrainte de choisir. Elle mobilise sa troisième solution provisoire "skikk og bruk»: savoir-vivre, puis soudain inspirée, l'échange contre "Etikette». La raison de ce choix nous est révélée ailleurs dans ses données TAPs:

Hva er det den boken til Randi Brattholm het? Var det ikke Etikette? Skikk og bruk? Nei, skikk og bruk, det blir litt for... Jeg kan jo blande litt, bruke litt gode manerer og litt... Ja, det går nok bedre når jeg kommer inn i det, så er den grei.
Comment s'intitulait le livre de Randi Brattholm? N'était-ce Etikette? Skikk og bruk? Non, le savoir-vivre, ça fait un peu trop... Mais je peux mélanger un peu, mettre bonnes manières ici, et là...Ça ira sans doute mieux dès que je m'y serai trempée, ça ira alors tout seul.

Comme c'était le cas pour Anne, Christine ressent le besoin de s'imprégner du texte de départ avant de se lancer dans la reformulation. Il est intéréssant de constater que la solution s'est présentée à elle par voie d'association avec le livre de Randi Brattholm, dont elle cherche à se remémorer le titre. C'est dans cette ligne de réflexion contextuelle associative qu'il me semble logique de situer la solution «skikk og bruk» retenue pour traduire BSAM. Cette locution visiblement en état de latence, est activée au moment où Christine cherche à vêtir d'une forme linguistique acceptable en norvégien la notion du comportement social au sein de l'entreprise véhiculé par le sigle français.

\section{$3.3[\ldots]$ se sont faits à la force du poignet [...]}

Présentons enfin l'expression figée «se sont faits à la force du poignet» dans l'énoncé (14) : Beaucoup de mes clients se sont faits à la force du poignet [...] 
L'expression figée, telle qu'elle est insérée dans le contexte cognitif de l'ascension sociale, nous permet de visualiser les clients d'Hélène Choumiloff comme des battants, qui partant de zéro ont réussi, grâce à leur détermination, à gravir les échelons de la hiérarchie, à monter dans l'échelle sociale. Le mouvement est vertical, du bas vers le haut.

\subsubsection{Comparaison de «se sont faits à la force du poignet» dans (14) aux solu- tions retenues par Anne (35), Brigitte (36) et Christine (37):}

(35) Anne :

Mange av mine klienter har albuet seg frem [...]

Beaucoup de mes clients ont joué des coudes pour avancer [...]

(36) Brigitte :

Mange av mine kunder har slått seg frem her i livet [...]

Beaucoup de mes clients se sont frayé un passage dans la vie [...]

(37) Christine:

Mange av mine kunder har jobbet seg oppover [...]

Beaucoup de mes clients se sont élevés par le travail [...]

Dans leur traduction, Anne, Brigitte et Christine restituent l'idée de détermination et de combativité par des métaphores autres que celle de l'original. Anne conceptualise manifestement la scène des coudes pour avancer, Brigitte celle des coups de poing. Bien que les mouvements ici soient plutôt de nature horizontale, il n'en reste pas moins que l'idée évoquée rejoint parfaitement celle des battants dans l'énoncé original. Quant à Christine, elle évoque l'idée de s'avancer par le travail dans la société par un mouvement vertical, comme c'était le cas dans l'énoncé original. Les trois créations discursives nous permettent de visualiser les clients d'Hélène Choumiloff comme des personnalités pugnaces. Examinons à présent de plus près les données processuelles de Brigitte.

\subsubsection{Analyse des données processuelles de Brigitte}

(38) Commentaires relevés dans son protocole de verbalisation

\begin{tabular}{|c|c|}
\hline Commentaires verbaux de Brigitte & Traduction française \\
\hline $\begin{array}{l}\text { Ja... det er også et litt sånn der bloms- } \\
\text { trende uttrykk: se faire à la force du } \\
\text { poignet. Det betyr vel at de har slått seg } \\
\text { fram her i livet på en måte. (Tenker) Det } \\
\text { blir en sånn self made man, det skal jeg } \\
\text { da ikke bruke, tror jeg, men... (Stryker } \\
\text { seg over haken) Ja, men vi får se. } \\
\text { (Skriver) Eeeh, client, skal man bruke } \\
\text { kunde eller klient her? Jeg er frista til å } \\
\text { bruke klient, jeg, eh kanskje...fordi at det } \\
\text { er et personlig forhold til de, på samme } \\
\text { måte som en advokat har til, har til sine } \\
\text { kunder og..., de kaller jo sine kunder for } \\
\text { klienter. Så jeg setter klient i første } \\
\text { omgang, så får jeg se på sammenhengen. }\end{array}$ & $\begin{array}{l}\text { Oui... voici une expression plutôt } \\
\text { imagée: se faire à la force du poignet, qui } \\
\text { doit vouloir dire qu'ils ont dû se frayer } \\
\text { un passage dans la vie en quelque sorte. } \\
\text { (Réfléchit) C'est une sorte de self made } \\
\text { man, ce n'est pas du tout ce que je vais } \\
\text { mettre, bien que... (Passe sa main sur } \\
\text { son menton) C'est à revoir. (Ecrit) Eeeh, } \\
\text { client, faut-il mettre client ou clientèle } \\
\text { ici? J'ai envie de mettre clientèle, eh peut- } \\
\text { être... parce qu'il est question d'une } \\
\text { relation personnelle, comme celle qu'a un } \\
\text { avocat envers, envers ses clients et..., ils } \\
\text { disent bien clients pour référer à leur } \\
\text { clientèle. Je vais donc mettre clientèle }\end{array}$ \\
\hline
\end{tabular}


Ja er det her...se sont faits à la force du poignet...(Klør seg i hodet) Har slått seg frem i livet. Jeg bruker det i første omgang... fordi det avspeiler dette med at poignet... (Gestikulerer) (Skriver) pour commencer, je verrai ensuite en fonction du contexte. Voyons se faire à la force $d u$ poignet... (Se gratte la tête) Se sont frayé un passage dans la vie. Je vais mettre ça pour commencer ... parce que ça reflète que le poignet... (Gesticule) (Écrit)

Brigitte remarque la motivation dans l'expression imagée de l'original: «se faire à la force du poignet ». La solution de transfert qui lui vient immédiatement à l'esprit ne sera pas mise en cause dans les trois révisions du texte qu'elle effectuera et est par ailleurs identique à sa solution finale. Bien qu'elle s'accorde un temps de réflexion aussitôt après avoir verbalisé sa solution de transfert, et qu'elle se l'explique en référant à une expression anglaise analogue qu'elle dit ne pas vouloir utiliser, avant de modérer quelque peu ce parti pris, elle décide de conserver sa solution initiale. La raison de ce choix saute aux yeux: elle perçoit manifestement un rapport conceptuel entre la façon d'avancer à la force du poignet dans le texte de départ et celle d'asséner des coups de poing pour franchir les obstacles rencontrés en cours de route dans l'expression norvégienne. En d'autres termes, consciente que le simple transfert des traits saillants de l'expression figée française nuirait à l'intelligibilité de sa traduction, Brigitte évoque une expression figée équivalente de sa mémoire. Si dans la solution retenue «slå seg frem i livet»: se frayer un passage dans la vie, aucune partie du bras n'est mentionnée, il n'en reste pas moins que la référence implicite aux poings est d'emblée perçue par le lecteur du texte d'arrivée.

(39) Donnés Translog de Brigitte

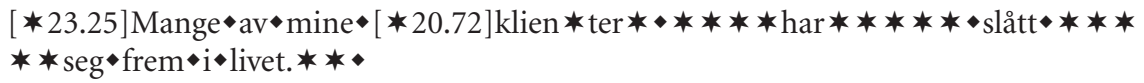

Beaucoup de mes clients se sont frayés un passage dans la vie.

La solution (39) alignée par Brigitte est identique à sa solution finale (36). La pause initiale de 23,35 secondes coïncide avec ses remarques sur le sens de l'expression française imagée et la création discursive qu'elle retient. La deuxième pause de 20,72 secondes est consacrée à la façon de traduire "client». Ses commentaires à ce sujet, bien qu'intéressants ne seront pas analysés dans le cadre de cette étude. En revanche ce qui importe ici est de faire ressortir que Brigitte ne se pose pas la question de savoir comment traduire l'expression figée. Elle aligne sans effort apparent la première solution qui lui est spontanément venue à l'esprit.

\subsubsection{Analyse des données de Brigitte}

Si la dimension métaphorique dans l'énoncé original (14) est conservée dans la traduction de Brigitte, les traits saillants qu'elle retient pour désigner la notion de réussite professionnelle au moyen d'efforts soutenus ne sont pas les mêmes que ceux de l'original. La création d'une nouvelle synecdoque est visiblement due à sa conceptualisation de la scène où les clients combatifs d'Hélène Choumiloff franchissent les obstacles rencontrés en cours de route en se servant de leurs poings. 


\section{Trois catégories de procédure de transfert}

Nous avons vu que les deux expressions inédites (12) et (13) et l'expression figée (14) ont donné lieu à des créations discursives dans tous les cas, sauf un : la solution de Brigitte au sujet de BSAM. L'analyse conduite à leur égard me fait percevoir trois grandes catégories de procédures de transfert - qui seront bien entendu complétées et affinées au fur et à mesure que l'étude avance - selon que les informations sont convoquées ou évoquées (Delisle 1980: 81) par le traducteur. Il s'agit des procédures de transfert:

1. Automatique dans le sens de routinier où le traducteur convoque des structures linguistiques à partir de schèmes cognitifs de sa mémoire sans efforts apparents. À force d'avoir été répétés, ces structures et ces schèmes, même s'ils sont complexes, sont parfaitement maîtrisés. Automatique est donc compris ici dans un sens analogue à celui qu'attribue Langacker à automation:

Automation occurs when a linguistic structure or a knowledge frame is mastered quite thoroughly, to the extent it can be employed in a largely automatic fashion. Through repetition a complex structure comes to be manipulated as a "prepacked assembly» and automation has occurred (Langacker 1987: 57).

2. Exploratoire dans le sens où le raccordement des concepts aux formes linguistiques s'opère par voie d'association. Le traducteur évoque une solution de façon plus ou moins instantanée. Le surcroît de réflexion correspond à un va-et-vient entre le sens immatérialisé et les formes linguistiques susceptibles de restituer celui-ci dans la langue d'arrivée, ce va-et-vient pouvant donner lieu à plus d'une solution intermédiaire.

3. Réfléchie, car soumise à un discernement. Cette procédure de transfert se situe à cheval entre les deux procédures de transfert précédentes. Le traducteur évoque par association des schèmes cognitifs de sa mémoire, lesquels donnent spontanément lieu à des formulations linguistiques.

Pour terminer, tâchons de classer la démarche des experts-traducteurs en fonction de ces trois catégories.

\section{1. [...] balayées par l'ouragan post-soixante huitard [...]}

J'interprète les commentaires verbaux d'Anne sur la nécessité de s'imprégner du texte de départ avant de se lancer dans la reformulation, ainsi que la durée moyennement importante des deux pauses intervenant avant et après avoir écrit «soixante-huit» et "génération", à savoir respectivement 20,78 et 11 secondes, lesquelles ne coïncident pas avec une importante verbalisation, signe d'un effort cognitif certain (voir la parcimonie de ses commentaires verbaux dans (18) ci-dessus), comme des indices d'un effort cognitif soutenu, homologuant une procédure de transfert exploratoire.

\subsection{Le «BSAM» (bonjour-sourire-au-revoir-merci) [...]}

J'infère à partir des données processuelles de Christine une démarche similaire à celle d'Anne. Bien que la solution adoptée pourrait être interprétée comme le fruit d'une procédure de transfert automatique en raison de la courte pause de 4 secondes, celle-ci 
ne doit pas être vue isolément, mais au contraire mise en rapport avec les commentaires verbaux de Christine au sujet de la façon de traduire bonnes manières dans le titre du texte, ainsi qu'avec les pauses enregistrées par le logiciel Translog coïncidant avec ces commentaires. Rappelons que la décision de Christine avait fait l'objet d'une mûre réflexion. Pour ces raisons, je choisis de classer sa solution comme une procédure de transfert exploratoire, le transfert effectué au moyen d'une création discursive s'étant produit moyennant un effort conscient de reformulation, avec plusieurs vaet-vient entre le sens immatérialisé et les diverses formes linguistiques retenues pour restituer celui-ci dans la langue d'arrivée.

\section{3. [...] se sont faits à la force du poignet}

Pour ce qui est des données processuelles de Brigitte, la solution retenue semble découler de l'évocation d'une scène cognitive qui donne lieu à la création spontanée d'une formulation linguistique. Je classe ainsi la démarche de Brigitte dans la catégorie d'une procédure de transfert réfléchie. Bien que la pause initiale de 23,35 secondes soit moyennement importante, elle coïncide avec une verbalisation importante (voir (27) ci-dessus) où Brigitte suggère d'emblée la solution: se frayer un passage dans la vie, qu'elle associe aussitôt à une expression anglaise équivalente. Sa découverte quasi immédiate d'une synecdoque de même poids stylistique que l'original semble découler d'une inspiration fondée sur « une compréhension parfaite des idées à rendre alliée à une disponibilité totale des moyens linguistiques pour les exprimer » (Delisle 1981: 82). Les trois pauses de 9,5 et 5 secondes qui précèdent l'alignement de sa création discursive coïncident avec la suite de son raisonnement (voir ses commentaires verbaux dans (30) ci-dessus) où elle se demande s'il lui faudrait peut-être poursuivre la piste du "self made man», solution intermédiaire qu'elle abandonne cependant au profit de sa solution initiale. Nous relevons un souci chez Brigitte de valider son choix initial au moyen d'une comparaison avec une solution intermédiaire considérée comme inférieure à sa solution initiale.

L'analyse qualitative de ces trois expressions linguistiques a permis de montrer que les experts-traducteurs ont eu recours à des procédures de transfert exploratoires et réfléchies pour traduire aussi bien les expressions inédites que figées dans la langue d'arrivée, celles-ci donnant lieu à la création de nouvelles synecdoques. Il va cependant de soi qu'une analyse aussi restreinte ne permet aucunement de corroborer ou d'infirmer l'hypothèse selon laquelle les mêmes mécanismes cognitifs sont à l'œuvre pour traduire les deux types d'expressions linguistiques. L'analyse des données empiriques relatives aux autres locutions de l'ensemble du texte, permettront, espérons-le, de faire le point sur la nature des mécanismes cognitifs à l'œuvre chez le professionnel expérienté de la traduction.

\section{Conclusion}

Essayer de cerner la démarche traduisante en se fondant sur les données processuelles in vivo des sujets traduisants est un travail passionnant, mais à la fois minutieux et de longue haleine. La visée à terme est d'être en mesure de proposer des explications probantes aux indices d'activités cognitives représentées par les données linguistiques, temporelles et comportementales des sujets traduisants. 
Comme l'objectif de la linguistique cognitive est de trouver une explication aux activités cognitives de l'être humain aux prises tant avec la conceptualisation qu'avec la recherche d'une formulation susceptible de représenter le concept, je suis convaincue qu'elle se prête particulièrement bien aux études sur la traduction. Autant que je sache, aucune théorie linguistique n'a su rendre compte d'un aspect primordial en traduction: celui de la créativité, lequel est étroitement relié au comment de la compréhension et de la production du sens. Il est à espérer que les recherches en linguistique cognitive permettront d'éclairer ces phénomènes fondamentaux de la communication humaine, qui constituent l'essence même de la traduction.

Je suis d'autre part persuadée que la traduction peut, de son côté, servir la linguistique cognitive. Du fait que l'étude du processus traductionnel offre un excellent poste d'observation au phénomène du sens - puisqu'elle permet d'analyser celui-ci sous ses deux aspects: celui de la compréhension et celui de la reformulation - la traduction fournit à la linguistique cognitive des données empiriques précieuses fondées sur des discours authentiques dans plusieurs langues à partir desquelles elle peut non seulement tester ses hypothèses sur les processus cognitifs, mais aussi proposer de nouvelles hypothèses falsifiables.

\section{NOTES}

1. La présente étude s'inscrit dans un programme de recherche plus vaste sur les processus cognitifs du traducteur intitulé: La traduction: point de contact entre langues et cultures, financé par le Conseil norvégien de recherche scientifique pour la période 2001-2005.

2. Seules les données de ces trois experts-traducteurs sont analysées dans le cadre du présent article, les données des autres sujets traduisants participant à l'expérience (jeunes professionnels de la traduction avec deux ans d'expérience ainsi que des non professionnels de la traduction) sont analysées ailleurs (voir Rydning 2004).

3. Pour une description des caractéristiques du passif en norvégien, voir Norsk Referansegrammatikk 1995: 837-852.

\section{RÉFÉRENCES}

Barcelona, A. (ed.) (2000): Metaphor and Metonymy at the Crossroads - A cognitive Perspective, Berlin/New York, Mouton de Gruyter.

Bereiter, C. and M. Scardamaglia (1987): The Psychology of Written Composition, New Jersey/ London, Hillsdale.

Deslisle, J. (1980): L'analyse du discours comme méthode de traduction, Ottawa, Éditions de l'Université d'Ottawa.

Deslisle, J. (1993): La traduction raisonnée. Ottawa, Éditions de l'Université d'Ottawa.

Feyaerts, K. (2000): Refining the Inheritance Hypothesis. Barcelona, A. (ed.): Metaphor and Metonymy at the Crossroads. Berlin/New York, Mouton de Gruyter, p. 59-78.

Gibbs, R. W. (1994): The Poetics of Mind. Cambridge: Cambridge University Press.

Gibbs, R. W. and G. Steen (1999): Introduction in Gibbs, R.W. and G. Steen (eds): Metaphor in Cognitive Linguistics 1-8, Amsterdam/Philadelphia, John Benjamins.

JäÄskeläInen, R. (1987): What Happens in a Translation Process: Think-aloud Protocols of Translation, Joensuu, University of Joensuu, Savonlinna School of Translation Studies.

JäÄskeläınen, R. (1989): Translation Assignment in Professional vs. Non-professional Translation: A Think-aloud Protocol Study, in SÉGuinot, C. (ed.): The Translation Process. Toronto, H.G. Publications, p. 87-98.

JäÄskelärnen, R. (1990): Features of Successful Translation Processes: A Think-aloud Protocol Study, Joensuu, University of Joensuu, Savonlinna School of Translation Studies. 
JäÄskeläInen, R. (1998): Think-aloud Protocols, in BAKeR, M. (ed.) : Routledge Encyclopedia of Translation Studies, London/New York, Routledge, p. 265-269.

JäÄskeläInen, R. (2000): Tapping the Process - An Explorative Study of the Cognitive and Affective Factors Involved in Translating, Joensuu, University of Joensuu, Savonlinna School of Translation Studies.

Jakobsen, A.L. (1999) : Logging target text production with Translog, Hansen, G. (ed.) : Probing the Process in Translation: Methods and Results Copenhagen Studies in Language 24, Copenhagen, Samfundslitteratur, p. 9-20.

Jakobsen, A.L. and L. Schou (1999): Translog documentation in Hansen (ed.): Probing the Process in Translation: Methods and Results Copenhagen Studies in Language 24, Copenhagen, Samfundslitteratur, p. 73-101.

Jakobsen, A.L. (2000): Understanding the Process of Translation: The Contribution of TimeDelay Studies, in Dimitrova, B.E. (ed.): Översättning och tolkning, p. 155-172.

Jensen, A. (2000): The Effects of Time on Cognitive Processes and Strategies in Translation, Doctoral dissertation Ph.D., Copenhagen, Copenhagen Business School.

Lakoff, G. and M. Johnson (1980): Metaphors We Live By, Chicago/London, The University of Chicago Press.

Lakoff, G. and M. Turner (1988): More than Cool Reason, Chicago/London, The University of Chicago Press.

LANGACKeR, R.W. (1987): Foundations of cognitive Grammar, Stanford, Stanford University Press.

Langacker, R.W. (1991): Concept, Image, and Symbol - The Cognitive Basis of Grammar, Berlin and New York: Mouton de Gruyter.

Lederer, M. (1976): «Synecdoque et traduction», in Seleskovitch (ed.): Traduire: Les idées et les mots. Paris: Etude de linguistique appliquée 24, 13-41.

Lederer, M. (1981): La traduction simultanée - Fondements théoriques, Paris, Minard.

LEDERER, M. (1994): La traduction aujourd'hui - Le modèle interprétatif, Paris, Hachette.

Norsk Referansegramatikk (1997): Faarlund, J-T., Lie, S. et Vannebo, K-I. Oslo: Universitetsforlaget.

Panther, K-U. and G. Radden (eds) (1999): Metonymy in Language and Thought. Amsterdam/ Philadelphia: John Benjamins Publishing Company.

Poulsen, S. (1992): Kollokasjonsundersøkelse inden for engelsk merkantilt og økonomisk sprog med henblik på uadarbejdelse af ordbogskoncept. Fjeld, R.V. (ed.): Nordiske studier $i$ leksikografi. Rapport fra konferanse om leksikografi i Norden 28 - 31 mai 1991. 288-295. Oslo : Nordisk forening for leksikografi.

Reboul, O. (1994): Introduction à la rhétorique, Paris, Presses universitaires de France.

RYDNing, A.F. (2000): TAPs (Think-aloud protocols) - A useful method in throwing light on the translation process. Dørum, H., Nilsson, K. et Schult Ulriksen, S. (eds) : Romansk Forum 11 : 91-110. Oslo: Université d'Oslo.

Rydning, A.F. (2001a.): La synecdoque - concept clé en traduction. Dørum, H., Nilsson, K. et Schult Ulriksen, S. (eds): Romansk Forum 13: 19-41. Oslo: Université d'Oslo.

Rydning, A.F. (2001b.): The synecdochial device applied to translation seen against the conceptual metaphor theory (CMT). Dørum, H., Nilsson, K. et Schult Ulriksen, S. (eds) : Romansk Forum 14: 61-73. Oslo: Université d'Oslo.

Rydning, A.F. (2003) : La métonymie conceptuelle. Dørum, H., Nilsson, K. et Schult Ulriksen, S. (eds): Romansk Forum 17: 71-84. Oslo: Université d'Oslo.

Rydning, A.F. (2004) (sous presse) : Etude de l'effort cognitif du traducteur lié à la reformulation de métaphores. Israël, F. et Lederer, M. (eds.): La Théorie interprétative de la traduction: regards croisés, Paris: Minard Lettres Modernes (28 pages).

RYdning, A.F. (2005): (sous presse) Creativity in translation in the light of the cognitive blending theory (BT). Lederer, M. et Jungwha Choi (eds). Forum, vol. 3, n 1, 2005. Paris: Presses de la Sorbonne Nouvelle (25 pages). 
Seto, K.-I. (1999) : Distinguishing Metonymy from Synecdoche. Panther, K-U. et Radden, G. (eds) : Metonymy in Language and Thought. Amsterdam/Philadelphia: John Benjamins Publishing Company.

Tirkkonen-Condit, S. and J. Laffling (eds.) (1993): Recent Trends in Empirical Translation Research. Joensuu: University of Joensuu.

Tirkkonen-Condit, S. (1989): Professional vs. Non-professional Translation: A Think-aloud Protocol Study. Séguinot, C. (ed.): The Translation Process. Toronto. H.G. Publications.

Tirkkonen-Condit, S. (1992): The Interaction of World Knowledge and Linguistic Knowledge in the Process of Translation. A Think-aloud Protocol Study. Lewandowska-Tomaszczyk, B. et M. Thelen (eds): Translation and Meaning, Part 2 Maastricht: Rijkshogschool Maastricht, Faculty of Translation and Interpreting. 